http://jmscr.igmpublication.org/home/ ISSN (e)-2347-176x ISSN (p) 2455-0450

crossref DOI: https://dx.doi.org/10.18535/jmscr/v10i2.28

Journal Of Medical Science And Clinical Research

IGM Publication

An official Publication of IGM Publication

\title{
A Prospective Study to Compare Chemotherapy and Radiotherapy in Palliative Treatment of Locally Advanced Carcinoma Gall Bladder
}

\author{
Authors \\ Avinash Ranjan", S.N. Prasad ${ }^{2}$, Jitendra Verma ${ }^{3}$, Pramod Kr Singh ${ }^{4}$, \\ Archana Singh ${ }^{5}$, Md. Waseem Raza ${ }^{6}$ \\ ${ }^{1}$ Junior Resident IIIrd Year, ${ }^{2} \mathrm{HOD}$ and Professor, ${ }^{3,4}$ Associate Professor, ${ }^{5,6}$ Assistant Professor \\ Department of Radiation Oncology J.K. Cancer Institute, Kanpur, Uttar Pradesh, India
}

\begin{abstract}
Background: Currently, there is no standard therapy for advanced gallbladder cancer. In patients who are beyond the scope of curative treatment at the time of diagnosis, palliative chemotherapy and palliative radiotherapy can be prescribed but the survival is counted in months and the quality of life is further hampered due to toxicity of drugs.

Objective: With respect to response and acute toxicities we evaluated the efficacy and toxicities of radiotherapy and chemotherapy in three arms. We compared the loco regional response in 3 arms after completion of treatment and at the end of 2 months and 6 months of radiotherapy and chemotherapy.

Material and Methods: Sixty patients of surgically unresectable locally advanced carcinoma gall bladder were enrolled. They were randomized in three arms. Arm A received radiotherapy 30 Gy in 10 fractions, Arm B received radiotherapy 20 Gy in 5 fractions and Arm C received chemotherapy Inj gemcitabine with Inj cisplatin for 6 cycles.

Results: Median follow up of all arms were 8 months. 8 months progression free survival (22\% vs $10.5 \%$ vs $29.4 \%$ p value-0.2010) and overall survival (54\% vs $42 \%$ vs 51\% p value-0.08) were comparable in 3 arms. Hematological toxicities, nausea and vomiting were significantly greater in chemotherapy arm. Locoregional response and QOL were comparable in all 3 arms.

Conclusion: Palliative radiotherapy had comparable locoregional response with significantly lower toxicity profile. Thus, palliative radiotherapy can be considered as an alternative to chemotherapy in treatment of locally advanced carcinoma gall bladder.

Keyword: Gall bladder cancer, palliative chemotherapy, palliative radiotherapy, Inj gemcitabine, Inj cisplatin, locoregional response.
\end{abstract}

\section{Introduction}

Carcinoma gall bladder is the most common malignant tumor of the biliary tract and the fifth commonest among malignant neoplasm of the gastro intestinal tract and its incidence has been increasing in recent years.
As per GLOBOCAN 2019, the worldwide age standardized incidence and mortality (per 1,00,000) for GBC are 2.3 and 1.7, respectively.

Approximately $50-60 \%$ of the non metastatic GBC present at a locally advanced stage (T3/T4 or $\mathrm{N}+$ ). The median survival for locally advanced gall 
bladder cancer (LAGBC) in multiple series ranges from 6-16 months with a 2 year survival of $<20 \%$. Resection is the most effective and only potentially curative treatment. Early-stage tumors are often curable with a proper resection; however, many patients present late in the course of the disease when surgical intervention is no longer effective. Patients with unresectable or metastatic gall bladder cancer have a poor prognosis. In patients with suspected gall bladder cancer, an open surgical resection is advocated. Adjuvant combination chemotherapy and molecular targeted therapy are emerging as effective therapeutic options in those with advanced gall bladder cancer. Endoscopic palliation of biliary and gastric outlet obstruction with metallic stents has improved the quality of life. Currently, there is no standard therapy for advanced gallbladder cancer. In patients who are beyond the scope of curative treatment at the time of diagnosis, palliative chemotherapy can be prescribed but the survival is counted in months and the quality of life is further hampered due to toxicity of drugs.

Present study is a prospective analysis to evaluate the role of palliative chemo-radiotherapy in advanced carcinoma gall bladder where no satisfactory treatment is available and patient is generally sent home on symptomatic treatment. Resection of gross disease is usually not possible due to late diagnosis and there is no definitive role of radiation or chemotherapy for cure.

\section{Material and Methods}

This study is a prospective analysis of patients of Locally Advanced Carcinoma Gall Bladder patients treated between November 2019 to July 2021 at J.K. cancer institute. Ethics Committee Board approval was obtained for the study.

\section{Inclusion Criteria}

Based on the above assessment the patients for the studywas selected depending on the following criteria:

$>$ Histologically proven cases of carcinoma gall bladder presenting to OPD of J.K. Cancer Institute, Kanpur

Karnofsky Performance Status $\geq 70$.
Complete hemogram with $\mathrm{Hb}>10 \mathrm{gm} / \mathrm{dL}$; TLC $>4000 / \mathrm{cmm}$, Platelet count $>100,000 / \mathrm{cmm}$

$>$ Renal function tests with Blood urea < $40 \mathrm{mg} / \mathrm{dL}$ and Serum creatinine $<1.5 \mathrm{mg} / \mathrm{dL}$.

$>$ Inoperable advanced carcinoma gall bladder.

$>$ Patients who sign the informed consent and are ready to be on follow up as required.

\section{Exclusion Criteria}

The patients having any of the following conditions will be excluded from the study:

$>\quad$ Post operated carcinoma gall bladder

$>$ Prior radiation or chemotherapy for the disease.

$>$ A poor general condition with Karnofsky performance Status of $<70$.

$>\quad$ Pregnant or lactating patient

\section{Methodology}

$>$ Patients fulfilling the above inclusion criteria with cancer gall bladder reporting to J.K. Cancer Institute, Kanpur Nov 2019 to July 2021 was randomly divided into three groups.

Arm A: Radiotherapy alone (3000 cGy in 10\#, D1D10 (2 wks) 5 fractions per week by DRR.

Arm B: Radiotherapy alone (2000 cGy in 5\#, D1D5 (1 wks) 5 fractions per week by DRR.

Arm C : Chemotherapy alone gemcitabine 1000 $\mathrm{mg} / \mathrm{m} 2$ (day 1 , day 8 ) + cisplatin $75 \mathrm{mg} / \mathrm{m} 2$ day 1 every 21 days for 6 cycles.

\section{Follow-up/ Toxicity}

All the patient was followed up regularly on OPD basis once every 15 days after completion of the treatment. At every visit, each patient was clinically evaluated for local control of disease and treatment related complications. The patient was assessed for any evidence of distant metastasis/disease progression during each follow up. To evaluate the distant metastasis detailed history pertaining to any symptoms was taken and general physical examination of patients was done. In case of suspicion, relevant investigations was done to rule out the presence of distant metastasis. The QOL was assessed on the day of completion of treatment and 
one month after completion of planned treatment using University of Washington QOL questionnaire. The results of the study regarding safety, tolerability, toxicity and response in all the groups was documented.

\section{Statistics}

The primary end point of the study was progression free survival (PFS). PFS was defined as the time interval in months between the date of diagnosis of malignancy and the date of clinical/ radiological progression or death. The secondary endpoint was overall survival (OS) and acute/ late toxicity. Chi square test was used to ascertain difference between categorical variables.

\section{Results}

In our study we enrolled a total of 60 patients, 20 patients in each Arms. Because of lack of awareness \& Covid-19 Pandemic total of 6 patients defaulted from treatment.

- Age wise distribution in Arm A was maximum in age group $>60$ years i.e. $10(50 \%)$ patients, followed by 40-49 years that is $4(20 \%)$ patients, $50-59$ year $3(15 \%)$ patients, 31-39 year $3(15 \%)$ patients. Whereas in Arm B, maximum is in the age group 50-59 years $10(50 \%)$ patients, followed by $>60$ years $5(25 \%)$ patients, 40 49 years $3(15 \%)$ patients, 31-39 years, $2(10 \%)$ patients. Whereas in Arm C, maximum is in the age group 40-49 yrs $12(60 \%)$ patients followed by 50-59 yrs $5(25 \%)$ patients, $>60$ yrs. $2(10 \%)$ patients, 31-39 yrs 1(5\%) patients. Overall maximum patients are in age group (40-59 years) that is $37(61.6 \%)$ patients.

- In Arm A there were maximum female 17 (85\%) patients and $3(15 \%)$ male patients whereas in Arm B, maximum were female $18(90 \%)$ patients and male $2(10 \%)$ patients, whereas in Arm $\mathrm{C}$ all patients were female.

- Residence wise distribution in Arm A was more from rural background i.e 14(70\%) than in urban i.e. 6(30\%) patients. In Arm B more patients were from rural background that is $16(80 \%)$ patients than in urban $4(20 \%)$ patients. In Arm $\mathrm{C}$ more patients were from rural background i.e. 14(70\%) patients than in urban i.e. $6(30 \%)$ patients.

- In our study in Arm A only 1 patient (5\%) have smoking habit whereas in Arm B 1 patient $(5 \%)$ was addicted to smoking and 1 patient $(5 \%)$ was addicted to alcohol, where in Arm C 1 patient (5\%) was addicted to smoking.

- In our study all patients were of KPS 70 \& 80. In Arm A \& B 10(50\%) patients were of KPS 70 and $10(50 \%)$ patients were of KPS 80. In Arm C 12 (60\%) patients were of KPS 70 and 8(40\%) patients were of KPS 80 .

- Our study have maximum patients of stage IV A \& IV B. In Arm A maximum patient was of stage IV A 8(40\%) patients followed by IVB $7(35 \%)$ patients followed by IIIB 4(20\%) patients. In Arm B maximum patients was of stage IVB $8(40 \%)$ patients followed by stage IVA $7(35 \%)$ patients followed by stage IIIB 4(20\%) patients. In Arm C maximum patients were of stage IVB \& IV A 8(40\%) patients each followed by IIIB 2(10\%) patients.

Median duration of treatment in Arm A \& B was very less as compared to Arm C. Arm A has median treatment duration of 14 days whereas Arm B has median treatment duration of 7 days \& Arm $\mathrm{C}$ has median treatment duration of 119 days.

Table No.1

\begin{tabular}{|l|c|c|c|}
\hline & A & B & C \\
\hline No. of patients & 20 & 20 & 20 \\
\hline Defaulters & 2 & 1 & 3 \\
\hline Stage III B & $20 \%$ & $20 \%$ & $20 \%$ \\
\hline Stage IV A & $40 \%$ & $35 \%$ & $40 \%$ \\
\hline Stage IV B & $35 \%$ & $40 \%$ & $40 \%$ \\
\hline Male & $15 \%$ & $10 \%$ & $0 \%$ \\
\hline Female & $85 \%$ & $90 \%$ & $100 \%$ \\
\hline KPS 80 & $50 \%$ & $50 \%$ & $40 \%$ \\
\hline KPS 70 & $50 \%$ & $50 \%$ & $60 \%$ \\
\hline $\begin{array}{l}\text { Median duration of } \\
\text { treatment }\end{array}$ & 14 days & 7 days & 119 days \\
\hline
\end{tabular}


- Hematological toxicity was more in Arm C than in Arm A \& B. In Arm A 6(33.33\%) patients had grade I Anemia, 1 (5.55\%) patient had grade II Anemia, whereas in Arm B 5(26.3\%) patients had grade I anemia, $1(5.2 \%)$ patients had grade II anemia. In Arm C 5(29.4\%) patients had grade I anemia $\&$ grade II anemia each whereas $2(11.76 \%)$ patients had grade 3 anemia. $x^{2}=11.92$, $\mathrm{p}=0.01$ (significant)

- Leucopenia was more in Arm $\mathrm{C}$ with 9(47.36\%) Grade-I, 4(21.05\%) patients Grade II and 1(5.88\%) patients grade III leucopenia. In Arm A 1(5.5\%) patients had grade I leucopenia. No patient had leucopenia in Arm B. $x 2=3.718 ; \mathrm{p}<0.001$; significant.

- Thrombocytopenia was more in Arm $\mathrm{C}$ that is 2 patients $(11.76 \%)$ patients Grade I thrombocytopenia. No patients had thrombocytopenia in Arm A \& B. $x^{2}=6.522$ $\mathrm{p}$ value $=0.03$ (significant) Hematological toxicity was significantly greater in Arm C.

- In our study $2(11.11 \%)$ patients have Grade I vomiting, $12(66.66 \%)$ patients have grade II vomiting, 4(22.22\%) patients have grade III vomiting in Arm A whereas in Arm B ; 6 (31.57\%) patients have grade I vomiting, 10 $(52.63 \%)$ patients have grade II $3(15.78 \%)$ patients have grade III vomiting. In Arm C; $7(41.17 \%)$ patients have grade II, $10(58.82 \%)$ patients have grade III vomiting. $\mathrm{x}^{2}=13.37$ $\mathrm{p}$ value $=0.009$ (significant)

- In Arm A $11(61.11 \%)$ patients have Grade I pain, 7 (38.88\%) patients had Grade II pain after completion of treatment. Whereas in Arm B $9(47.36 \%)$ patients had grade I pain and grade II pain each and $1(5.26 \%)$ had grade III pain. In Arm C 6(35.29\%) patients had grade I ; $9(52.99 \%)$ patients had grade II and $2(11.76 \%)$ patients have grade III pain. $\mathrm{x}^{2}=0.05 \mathrm{p}>0.05$ (not significant).
Table No. 2

\begin{tabular}{|l|c|c|c|}
\hline & A & B & C \\
\hline Anemia Grade I & $33.33 \%$ & $26.3 \%$ & $29.4 \%$ \\
\hline Anemia Grade II & $5.55 \%$ & $5.2 \%$ & $29.4 \%$ \\
\hline P value 0.01 & \\
\hline Leucopenia Grade I & $5.5 \%$ & $0 \%$ & $47.36 \%$ \\
\hline P - value < 0.001 & $0 \%$ & $11.76 \%$ \\
\hline $\begin{array}{l}\text { Thrombocytopenia } \\
\text { Grade I }\end{array}$ & $0 \%$ & $0 \%$ & \\
\hline P - value < 0.001 & & \\
\hline Vomiting Grade III & $22.2 \%$ & $15.78 \%$ & $58.88 \%$ \\
\hline P - value 0.009
\end{tabular}

- In Arm A 2(11.11\%) patients had very good QOL, 5(27.77\%) patients had good QOL, 7(38.88\%) patients had fair QOL 3(16.66\%) patients had poor QOL and $1(5.55 \%)$ patients had very poor QOL. In Arm B $1(5.26 \%)$ patients had very good QOL, $4(21.05 \%)$ patients had good QOL; $8(42.10 \%)$ patients had fair QOL, $4(21.05 \%)$ patients had poor QOL, 2(10.5\%) patients had very poor QOL. Whereas in Arm C $9(52.9 \%)$ patients had fair, $5(29.4 \%)$ patients had poor and $3(17.6 \%)$ patients had very poor QOL. $\mathrm{P}=0.23 \mathrm{x}^{2}=8.07$ (not significant)

- In our study at 2 month in Arm A $17(94.44 \%)$ patients had partial response and 1(5.55\%) patient had no response. In Arm B $17(94.44 \%)$ patients had partial response and $2(10.52 \%)$ had no response whereas in Arm C 14(82.35\%) had partial response and 3 (17.64\%) patients had no response. $\mathrm{x}^{2}=1.304 ; \mathrm{p}=0.52$ (not significant)

- In our study at 6 month in Arm A $11(61.11 \%)$ patient had progressive disease, $5(27.77 \%)$ patients had stable disease and $2(11.11 \%)$ expired whereas in Arm B $11(57.89 \%)$ had progressive disease; $3(15.78 \%)$ had stable disease and 5(26.31\%) expired whereas in Arm C 6(35.29\%) had progressive disease ; $6(35.29 \%)$ had stable disease and 5(29.41\%) expired.

(Response were comparable in all 3 ARMs at 2 and 6 months after completion of treatment). 
- In our study, Arm A had 8 month PFS of $22 \%$; 6 month PFS of $44 \%$; 4 month PFS of $77 \% ; 2$ month PFS of $94 \%$ whereas in Arm B 8 month PFS of $10.5 \%$; 6 month PFS of $21 \%, 4$ month PFS of $57 \%$ and 2 month PFS of $84.2 \%$ whereas as in Arm C 8 months PFS of $29.4 \%$; 6 month PFS of $52.1 \%$; 4 month PFS of $94.1 \%$ and 2 month PFS of $100 \%$ seen. $x^{2}=4.563 ; p=0.60$ (not significant)

- Median PFS of Arm A is 6 months whereas of Arm B is 5 months whereas of Arm C is 7 months. PFS were comparable in all 3 ARMs. $\mathrm{x}^{2}=5.64 ; \mathrm{p}=0.076$; (not significant).

- 8 months OS of Arm A was $54 \%$ whereas 8 months OS of Arm B was $42 \%$ whereas 8 months $\mathrm{OS}$ of Arm $\mathrm{C}$ was 51\%. $\mathrm{P}=0.08$. (not significant).

Table No. 3

\begin{tabular}{|l|c|c|c|}
\hline $\begin{array}{l}\text { Response at 6 } \\
\text { month }\end{array}$ & A & B & C \\
\hline $\begin{array}{l}\text { Progressive } \\
\text { disease }\end{array}$ & $61.11 \%$ & $57.89 \%$ & $\begin{array}{c}35.29 \% \\
\text { P value } 0.08\end{array}$ \\
\hline Stable Disease & $27.7 \%$ & $15.7 \%$ & $\begin{array}{c}35.29 \% \\
\text { P value } 0.07\end{array}$ \\
\hline Expired & $11.11 \%$ & $26.3 \%$ & $\begin{array}{c}29.4 \% \\
\text { - value } 0.06\end{array}$ \\
\hline 8 months PFS & $22 \%$ & $10.5 \%$ & $\begin{array}{c}29.4 \% \\
\text { - value } 0.07\end{array}$ \\
\hline 6 months PFS & $44 \%$ & $21 \%$ & $\begin{array}{c}52.9 \% \\
\text { - value } 0.06\end{array}$ \\
\hline 4 months PFS & $77 \%$ & $57 \%$ & $\begin{array}{c}94.1 \% \\
\mathrm{P}-\text { value } 0.07 \\
7 \text { months } \\
\text { P value } 0.2\end{array}$ \\
\hline Median PFS & 6 & $\begin{array}{c}5 \\
\text { months }\end{array}$ & $\begin{array}{c}51 \% \text { P value } \\
0.08\end{array}$ \\
\hline 8 month OS & $54 \%$ & $44 \%$ & \\
\hline
\end{tabular}

\section{Discussion}

Over $80 \%$ Gall bladder cancers in India are diagnosed in advanced stage, leading to little chance of cure (Batra $Y$ et al 2005). Female illiteracy, gender bias and poor public health services contribute to late diagnosis.

N. Eleftheriadis et al (2001) presented a case of unresectable Gall Bladder carcinoma stage IV, histologically diagnosed was treated with external radiotherapy by $\mathrm{Co}^{60}$, using a three filed technique (anterior posteriors and R lateral) with the total dose of $3000 \mathrm{cGy}$ in 10\#, and concluded that external radiotherapy is a safe and effective method of palliative management of Gall Bladder Cancers.

Shwetabh Sinha et al (2020) analysed forty-five patients of surgically unresectable non-metastatic locally advanced Gall Bladder Cancer. Twenty patients did not receive Radiotherapy, while 25 patients received RT with conformal technique. At median follow up of 11.5 months, two year progression free survival ( $18.6 \%$ vs 0$)$ and overall survival $(37.3 \%$ vs $5 \%)$ were significantly better in RT cohort as compared to No RT cohort. Thus, they concluded that addition of local radiotherapy to chemotherapy improve survival outcome and can be considered as definite treatment modality for non metastatic LAGBC not amenable to surgery.

Valle $J$ et al (2010) enrolled 410 patients of advanced biliary tract cancer to receive CisplatinGemcitabine and Gemcitabine only. At median follow up of 8.2 month, median progression free survival was 8.0 month in cisplatin-gematabine group and 5.0 month in Gemcitabine only group. Our study had a similar progression free survival in chemotherapy arm.

A report of National Cancer Database of the USA of 11,190 gallbladder cases showed that receipt of radiotherapy was an independent predictor of improved OS in LAGBC. Similarly, in a SEER database review of 453 patients of biliary tract cancers (11\% GBC), patients who received RT had an improved survival (HR 0.82, 95\% CI:0.72-0.97, $\mathrm{p}=0.02$ ). Results of this population database analyses are in line with our results.

In our study maximum number of patients were between 40 to 60 years of age, female, belonged to rural background, had no addiction habits and were of stage IV. Majority of patients were of KPS 7080 .Arm A \& B have shorter treatment time than Arm C. Arm C have significantly greater incidence of Grade II \& grade III anemia. Arm C have significantly greater incidence of leucopenia. Arm C have significantly greater incidence of thrombocytopenia. Arm $\mathrm{C}$ had significantly greater severity of vomiting than Arm A \& B. Anorexia were comparable in all arms. Arm A \& Arm B have 
slightly better QOL than Arm C but data were not significant. There were comparable response in all three Arms at 2 months and 6 months. Median progression free survival were comparable in all three Arms at 2 months, 4 months, 6 months and 8 months. 8 months OS were comparable in all 3 arms.

\section{Conclusion}

Palliative radiotherapy $30 \mathrm{~Gy}$ in $10 \#$ and $20 \mathrm{~Gy}$ in $5 \#$ has a response, overall survival and progression free survival comparable to the palliative chemotherapy (Gemcitabine+cisplatin). Palliative radiotherapy have significantly lesser toxicity profile in term of hematological toxicity, nausea \& vomiting. Palliative radiotherapy have greater patient compliance than chemotherapy. Median treatment time is very less in palliative radiotherapy Arm than in chemotherapy Arm. Palliative Radiotherapy is well tolerated in maximum patient. Palliative radiotherapy resulted in better quality of life than in chemotherapy Arm but difference were not significant. Shortened treatment time in radiotherapy Arm lead to lesser economical burden on resources and more number of patients can be treated with the same resources. Palliative radiotherapy $30 \mathrm{GY} / 10$ \# had slightly better response at 2 month \& 6 months than in $20 \mathrm{~Gy} / 5 \#$ schedule. Progression free survival is also slightly better in 30 Gy/10\# Arm. But data were not significant. But the need of the hour is that studies with larger sample sizes and longer follow up should be instituted for further validation of the feasibility of palliative radiation and to get significant results so that we are able to consider palliative radiation in routine practice in case of advanced unresectable Carcinoma gallbladder.

\section{References}

1. Uno, T., et al. "Primary carcinoma of the gallbladder: role of external beam radiation therapy in patients with locally advanced tumor." Strahlentherapie und Onkologie: Organ der Deutschen Rontgengesellschaft... [et al] 172.9 (1996): 496-500.
2. Vij, Jagdish C., et al. "Endoscopic biliary endoprosthesis for palliation of gallbladder carcinoma." Gastrointestinal endoscopy 43.2 (1996): 121-123.

3. Houry, S., et al. "Gallbladder cancer: role of radiation therapy." Hepatogastroenterology 46.27 (1999): 1578-1584.

4. Donohue, John H. "Present status of the diagnosis and treatment of gallbladder carcinoma." Journal of Hepato-biliarypancreatic Surgery 8.6 (2001): 530-534.

5. Eleftheriadis, N., et al. "Is external palliative radiotherapy for gallbladder carcinoma effective?." Oncology Research and Treatment 24.6 (2001): 581-584.

6. Houry S, Barrier A, Huguier M (2001) Irradiation therapy for gallbladder carcinoma: recent advances $\mathbf{J}$ Hepatobiliary Pancreat Surg 8(6): 518- 524.

7. Batra, Yogesh, et al. "Gallbladder cancer in India: a dismal picture." Journal of gastroenterology and hepatology 20.2 (2005): 309-314

8. Ben-David MA, Griffith KA, Abu-Isa E, Lawrence TS, Knol J, et al. (2006) Externalbeam radiotherapy for localized extrahepatic cholangiocarcinoma. Int J Radiat Oncol Biol Phys 66(3):772-779.

9. Valle, Juan, et al. "Cisplatin plus gemcitabine versus gemcitabine for biliary tract cancer." New England Journal of Medicine 362.14 (2010): 1273-1281.

10. Sirohi B, Mitra A, Jagannath P, Singh A, Ramadvar M, et al. (2015) Neoadjuvant chemotherapy in patients with locally advanced gallbladder cancer. Future Oncol 11(10):1501-1509.

11. Henley SJ, Weir HK, Jim MA, Watson M, Richardson LC (2015) Gallbladder Cancer Incidence and Death Rates Cancer Epidemiology, Biomarkers and Prevention 24(9): 1319-1326.

12. Rishi Agarwal, Arun Sendilnathan, Nabeela Iffat Siddiqi, Shuchi Gulati, Abhimanyu Ghose, Changchun Xie, Olugbenga Olanrele 
Olowokure J Gastrointest Oncol. 2016 Dec; 7(6):996-1003. Doi: 10.21037/jgo.2016. 09.10

13. Schwartz LH, Litière $S$, de Vries E, Ford R, Gwyther S, et al. (2016) RECIST 1.1Update and Clarification: From the RECIST Committee. Eur J Cancer Oxf Engl 1990 62: 132-137.

14. JW Valle, I Borbath, SA Khan, F Huguet, T Gruenberger, et al. (2016) Biliary Cancer: ESMO Clinical Practice Guidelines 27: v28v37.

15. Verma V, Crane CH (2019) Contemporary perspectives on the use of radiation therapy for locally advanced gallbladder cancer. Chin Clin Oncol 8(4): 41-41.

16. Prashanth Rawla, Tagore Sunkara, Krishna Chaitanya Thandra, Adam Barsouk Clin Exp Hepatol. 2019 May; 5(2): 93-102. Published online 2019 May 23. doi: 10.5114/ceh. 2019.85166.

17. Shwetabh Sinha1, Reena Engineer, Vikas Ostwal, Anant Ramaswamy, Supriya Chopra, Nitin Shetty, et al (2020) Radiotherapy for Locally Advanced Unresectable Gallbladder Cancer-A Way Forward: Comparative Study of Chemotherapy Versus Chemoradiotherapy. 10.32474/OAJOM.2020.04.000177.

18. RTOG/EORTC Late Radiation Morbidity Scoring Schema. 